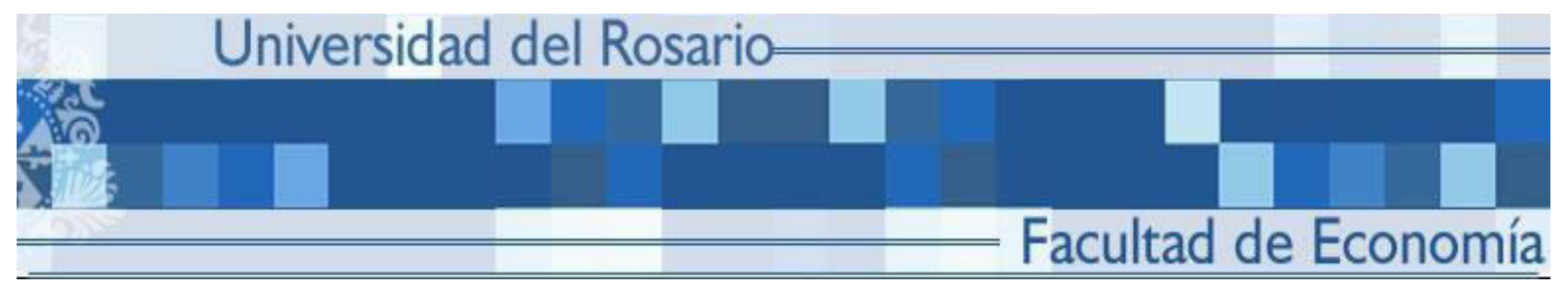

Does the market model provide a good counterfactual for event studies in finance?

Carlos Castro

SERIE DOCUMENTOS DE TRABAJO

No. 211

Diciembre de 2017 


\title{
Does the market model provide a good counterfactual for event studies in finance?
}

\author{
Carlos Castro ${ }^{1}$ \\ Faculty of Economics, Universidad del Rosario, Bogotá, Colombia
}

\begin{abstract}
We provide a common framework that relates traditional event study estimation methods in finance with a modern approach for causal event studies. This framework is called synthetic portfolio and is a particular case of synthetic control methods. We provide a simulation exercise and an empirical application to evaluate the performance of the method. In addition, synthetic control methods provides a reliable framework, for test based on the abnormal returns, that overcomes some difficulties in the traditional test. We conclude that the market model provides a counterfactual as good as a synthetic control.
\end{abstract}

Keywords: event studies, synthetic control methods, portfolio optimization, merger announcements.

JEL codes: G11, C13, G34.

\section{Introduction}

Event studies is one of the most widely used methodologies in accounting and financial research ([1]), and in certain legal proceedings. The timeline structure of an event study, determined by the estimation and event window has not changed dramatically since its introduction in the late sixties ([2]). There are important number of contributions that have focused specially on providing better tools for statistical inference, see [3] for a recent discussion. A recurrent element in event studies is the use of the market model to estimate the so call normal returns. In fact, [3] argues that the popularity of

\footnotetext{
${ }^{1}$ Corresponding author: carlos.castro@urosario.edu.co.
} 
event studies stems from a coincidence of developments in financial market research in the late 60s: CAPM, the CRSP data and more sophisticated and accessible statistical software. The author also concludes, that due to the size of research output using event studies published in the major finance journals, accounting journals and the use in other fields, the methodology continues to be popular and will continue to be an important element in empirical capital market research.

In the field of accounting in particular, and finance there is a recent interest in using the tools in empirical microeconomics to address classical issues in accounting research. The claim is that better research designs and statistical methods, in empirical microeconomics, have increased the credibility of the implications obtained in these studies. [4] provide an analysis of the use and potential of causal inference methods in the field of accounting research. Their main conclusions are as follows: accounting research does primarily address problems that are causal in nature; there is an increase in the use of quasi-experimental methods in addressing causal questions in accounting research; there is still a lot to be done in the field to use the tools that are already available and have been successfully used in empirical microeconomics; the authors emphasize the use of causal diagrams and structural models in accounting research. The potential outcome framework for causal inference has also been used in empirical finance in recent years. The potential outcomes are generally considered as missing variables in the causal inference literature because it is not possible to observe all the instances of the variable of interest simultaneously. In many financial event studies the researcher only observes the treated observation. Estimation of potential outcomes in observational studies is usually performed using one of the following techniques or a combination of some of them ([5]): model based-imputation, weighting, blocking and matching methods. In model based imputation, a model is build in order to predict the missing potential outcome of unit that is not treated. This is exactly what traditional event studies do when they define the normal return model as the constant return model or the market model. For the causal inference literature [5] model based imputation is not recommended to estimate treatment effects because a proper fit can only be accomplished by specifying the post-event outcomes. Weighting and blocking use different methods ( being propensity score one of the most popular) to combine the in- 
formation of the control units in order to build a proper conterfactual ${ }^{2}$. Using the propensity score achieves a balance between treated and control groups in order to estimate an unbiased treatment effect. Matching techniques find direct comparisons or matches for each unit. For a given treated unit with a particular value for the covariates, one searches for a control unit with similar values in the covariates. A distance metric is needed to implement a matching technique so as to asses the trade-off in choosing between different units and/or controls. The use of propensity score for balancing and estimating causal effects has already been used in event studies in finance for example [6] use propensity score matching to re-examine the long-run underperformed anomaly of stocks after seasoned equity offerings. The authors find that under-performance could be due to incorrect matching. Once issuers and non-issuers are matched using propensity score they find that under-performance is economically and statistically non-significant. [7] uses propensity score matching to adjust for selection bias when comparing public and private equity acquisitions. The author finds no significant difference in the premiums between what public acquires pay for acquisition relative to private equity acquirers (after controlling for target and deal characteristics). The results is a sharp contrast to established results. This small sample of results show that re-examining event studies in finance with different causal approaches can lead to different results.

The synthetic control method ([8]), has received a lot of attention in comparative case studies on different subjects: terrorism, natural disasters, tabacco control programs. As opposed to competing methods, synthetic control method's strength relies in the use of a combination of units to built a more objective comparison for the unit exposed to the intervention, rather than a choosing a single unit or a $A d$ hoc reference group. The authors advocate for the use of data drive procedures to build the reference group. The synthetic control method is a weighted average of the available control units, that makes explicit: the contribution of each unit to the counterfactual of interest and the similarities (or lack thereof) between the unit affected by the event or the intervention of interest and the synthetic control in terms of the preintervention outcomes and other predictors of post-intervention outcomes. More recently synthetic control methods have been the focus of intense re-

\footnotetext{
${ }^{2}$ The propensity score is the average unit assignment (for a particular treatment) probability for units that share the same specific characteristic
} 
search and it is considered as the most important innovation in the policy evaluation literature in the last 15 years according to [9]. The most recent literature has been addressing some limitations of the method: [10] and [11] provide generalization of the synthetic control method that address dimension reduction prior or during the estimation of the weights. In the former the author also illustrates the relationship between an interactive fixed effects model and synthetic controls methods under which Difference-in-Difference method is a special case. [11] also relaxes some of the restrictions imposed by the estimation of the synthetic controls. [12] and [13] propose complementary approaches to perform statistical inference on the estimated average treatment effects from synthetic control methods, these are important contribution because the limiting properties of the estimator were not known for a broad set of data generating process and testing was based on a placebo randomization. The developments of these methods has also motivated by the use in macroeconomic oriented research questions [14].

Synthetic matching techniques applied for event studies in finance are not common, we are only aware of their application in a recent paper, [15]. In this paper the authors measure the effect of personal connections on the returns of financial firms. The study is based on the connections of Timothy Geithner to different financial institutions prior to his nomination as Treasury Secretary at the end of 2008. The synthetic matching methodology is used as a complement to the usual approach in event studies of capturing the difference between a treatment and control group using for the latter the mean return model or the fitted market model. In addition we have an earlier paper using synthetic matching to measure the effectiveness of volatility actions with intra-day stock market data $([16])$

In this paper we provide a detailed analysis of the synthetic matching technique, which we denote as synthetic portfolio method. The notion of a synthetic portfolio provided a common framework that relates traditional event study techniques, in particular the use of the market model, and synthetic control method, which is has been gaining popularity in the causal event literature in recent years. A common framework is important so as to evaluate the benefits of new methodologies with regards to the traditional approach that has been in use sin the late sixties. We provide explain the framework, as series of estimators and their relationship to the traditional methods and more recent approaches, such a difference-in-difference which is a special case of synthetic portfolio. These alternative methods are able to 
handle the high-dimensional challenge brought by the large asset space in the US stock market. We provide simulation results to evaluate the performance of the different method and provide an empirical application using as event merger announcements. In addition this new methodological framework that we claim encompasses traditional approaches provides additional and valuable insights to perform statistical inference over the individual abnormal returns (the treatment effects) that was hardly possible with the traditional testing framework. This is made possible by the recent work on statistical inference for synthetic control methods by [12] and [13].

The simulations exercise evaluates how well does each of the method is able accommodate the evolution of the asset in question before in the estimation window and also how well does it estimate the true treatment effect. The results indicate that the performance of the market model and the synthetic portfolio approach are quite similar in terms of biases and variance.

The empirical application reexamines effects of merger announcements on the value of the firm in the short run, that is in the immediate days after the announcement. As in the literature these affects are measured along different sub-samples, for example, deals that affects publicly trades firms versus private, deals that are finances by stocks or cash. The results indicate that the estimated effects of the merger announcements are similar across the different estimation approaches, both in terms of the point estimates as well as the variance. In addition the introduction of a feasible testing framework for short term studies over each individual event provides a more through analysis of the effects so as to determine the cases where the effects are non existent versus the cases where the effect is strictly positive or strictly negative. This opens the possibility to explore empirically in a second stage the different determinants of the cumulative abnormal returns taking into account this significant variation. Overall both the simulation results and the empirical application indicate that the market model fairs well with respect to competing approaches that have surfaces within synthetic control methods. One of the reasons is that the market model is also a portfolio that tracts the asset of interest and is able to provide a reliable potential outcome, in particular when the beta of the assets tends to one, as one would expect. However, we believe that this is a particular property of the large sample of equities in the US and that some of these results might not be true in markets with very few liquid trades stocks where the synthetic portfolio approach might be a 
better option ${ }^{3}$. This is a subject of further research.

The paper is organized as follows, Section 2 discusses how the potential outcome approach can be introduced to the traditional event studies approach. Section 3 presents the synthetic portfolio approach. Section 4 presents the simulation exercise and the results comparing synthetic portfolio to traditional event studies. Section 5 discuss the data and empirical applications for merger announcements and seasoned equity offerings. Finally, Section 6 concludes.

\section{Potential outcomes in event studies}

Traditional Event studies in finance have a standard setup in terms of the the time leading up to the event and the outcome variable of interest, in many cases the holding period returns of the stock. Let $t=T_{0}$ denote the moment of time when the event takes place. If we are performing an event study on daily returns then is is customary to define the event window as an interval around the event $\left[T_{0}-d, T_{0}+d\right]$ where $d$ denotes the number of days around the event. For short term event studies $d$ is usually 10,5 or 1 day $(\mathrm{s})$. Accounting for a gap between the event window and the estimation window is also recommended. The reason for the gap is that noisy information regarding the event might become available to market participants some days before and therefore the stock price could start to deviate from some "normal" behavior in the month before the event. The gap is around a month, $m$, or two before the event window $\left[T_{0}-d-m, T_{0}-d\right)$. The estimation window has usually a length of one year (250 days to approximate the number of days in a calendar year) of market returns before the start of the gap window. To avoid any confusing notation from this point on we will consider two excluding time intervals (figure B.1): the estimation window $\left[T_{1}, T_{3}\right]$ and the event window $\left[T_{4}, T_{5}\right]$. Note that the event window is centered in the actual time of the event. For completeness the gap is the interval $\left(T_{3}, T_{4}\right)$.

A recurrent element in event studies is the use of the market model to estimate the so call normal returns. Let $R_{1, t}$ denote the holding period returns of the stock price of the firm that is affected by the event (without

\footnotetext{
${ }^{3}$ One of the complications of stock markets with very few assets is the over representation of one or more assets in the market index. This creates an endogeneity problem in the process of estimation a counterfactual using the market model, see [16].
} 
loss of generality firm 1 is the only firm affected by the event). There is no formal definition for the "normal" returns, however the implementation of an event study requires the researcher to disentangle the effects of two types of information on stock prices $([17])$ : the information that is specific to a firm (the event) and the information that is likely to affect stock prices marketwide (or a subset of interchangeable stocks). The disentanglement requires a way to control for the latter using the "normal" expected behavior of returns. In traditional event studies the most common approach to define the normal returns, is to use a market model or another factor model (FamaFrench three factor or Carhart four factor model) ${ }^{4}$. In finance, factor models are used in many application, and although there is an extensive literature, there is also an important discussion on the validity of the factors used to explain the cross section of returns $([18])$. Event studies consider mainly the one factor market model.

$$
R_{1, t}=\hat{\alpha}_{i}-\hat{\beta}_{i} R_{m, t}, t \in\left[T_{1}, T_{3}\right]
$$

where $R_{m, t}$ denotes the market return. The parameters of the market model are estimated using the information from the estimation window.

In traditional event studies ([19]), the effect of a particular event on a stockss price is measured by the abnormal returns (ARs).

$$
A R_{1, t}=R_{1, t}-E\left[R_{1, t} \mid R_{m, t}\right], t \in\left[T_{4}, T_{5}\right]
$$

where $R_{1, t}$ is the actual return and $E\left[R_{1, t} \mid R_{m, t}\right]$ is the expected normal return. In the market model, the normal return is given by, $E\left[R_{1, t} \mid R_{m, t}\right]=\hat{R}_{1, t}$, therefore the expected return is the fitted value obtained in 1 . The abnormal returns measure the effect of the event on the return of the firm that have been affected by that particular event ${ }^{5}$.Therefore the notion of normal returns tries to measure the expected behavior of the returns in the absence of the event.

\footnotetext{
${ }^{4}$ Another approach is the the constant mean model where the normal return is the time-series average return over the estimation window

${ }^{5}$ Abnormal returns can also be interpreted as the event-adjusted performance of a particular stock, that is, the difference between the observed performance and the "normal" expected performance. This definition is more closely related to the calendar-time approach used to investigate events of financial relevance.
} 
In order to determine a causal impact of the event on the performance of the stock, first, we must see the "normal" expected as a mechanism to provide a measure of the expected behavior of the returns in the absence of the event and hence, the market model is a framework that provides a model based potential outcome. Second, if we note that event studies in finance are observational studies rather than perfectly randomized experiments, then we can use the potential outcome approach (also known as the Rubin Causal Model) to come up with an identification strategy for causal event studies.

One of the key insights of the Rubin Causal Model is to think of potential outcomes as missing variables. In the event study we observe the returns before the event in the estimation window, but in the event window we only observed the returns that are already affected by the event $R_{1, t}^{I}:=R_{1, t}$ for $t \in\left[T_{4}, T_{5}\right]$. This is equivalent to the notion that the stock price of firm 1 in the event window is subject to a treatment and the treatment is the event. For example, if the event is a merger announcement then there are two firms directly affected by this event the acquiring firm and the target firm (for simplicity let firm 1 be only the acquire firm). Once the announcement happens or the event then we cannot observe the state of the world where this event did not take place, therefore the missing potential outcome is $R_{1, t}^{N}$; that is the returns of firm 1 in the event window if the event had not taken place. The effect of the event (or treatment) will be equivalent to the notion of abnormal returns,

$$
A R_{1, t}=R_{1, t}-R_{1, t}^{N}, t \in\left[T_{4}, T_{5}\right]
$$

As mentioned in the introduction the causal inference literature provides various identification strategies to estimate causal effects using the potential outcomes approach (see [9], for a recent survey) we will now only focus on synthetic matching techniques base on the synthetic control method proposed in $[8]$.

\section{Synthetic portfolio}

In the potential outcome approach we have units of analysis that are partitioned into a treatment and a control group; borrowing an ideal experimental setup from randomized control trials. For financial event studies we consider one firm that is affected by an event and the outcome variable, over which we want to measure the effect of the event is the returns of the stock 
of that firm. In addition we have a larger universe of stocks for other firms (other units of analysis) that are trading at the same time. These other firms as long as they are not directly or indirectly affected by the event of interest can be used to create a control group of firms. The fact that the firms in the control group are not affected by the event of interest is very important assumption in the potential outcome framework. The methodology we propose is to use the returns of the firms in the control group to build a synthetic portfolio and use the returns of this portfolio as a potential outcome or a measure of the "normal" returns; that is the returns that we would observe had the event not taken place.

We arrive at this synthetic portfolio by applying synthetic control methods introduced by [8] to the problem at hand. The authors propose a weighted average of the units of analysis in the control group as a way to come up with a synthetic counterfactual. The weights are obtained by using a minimum distance estimator applied to a series of restrictions on the outcome variable and a set of exogenous variables.

Again we let $R_{1, t}$ denote the return of the stock of interest where we want to measure the effect of the event (the stock that has been treated). Conversely, the synthetic portfolio is built using the other stocks (that are not involved in a similar event and that are trading during the same days) to replicate the performance of the security of interest. These set of stocks make up the control group, $\left(R_{2, t}, \ldots, R_{J, t}\right)$. The methodology is very simple since we only need to estimate $w_{j}^{*}$ required to estimate the effect of the intervention by solving the optimal tracking problem. Therefore we have to solve,

$$
\underset{w}{\operatorname{minimize}} \sum_{t=T_{1}}^{T_{3}}\left(R_{1, t}-\sum_{j=2}^{J} w_{j} R_{j, t}\right)^{2}
$$

for the estimation window $t \in\left[T_{1}, T_{3}\right)$. It is possible to include in this optimization problem restrictions on the estimated weights, for example nonnegativity constraints $\left(w_{j} \geq 0, j=2, \ldots, J\right)$, constant weights $\left(w_{j}=\bar{w}, j=\right.$ $2, \ldots, J)$ or that the weights sum to one $\left(\sum_{j=1}^{J} w_{j}=1\right)$. A proper tracking of the stock of interest $R_{1, t}$ in the estimation window $\left[T_{1}, T_{3}\right]$ would guarantee that the synthetic portfolio can provide a potential outcome for the latent variable $R_{1, t}^{N}$, in the event window $\left[T_{4}, T_{5}\right]$. The goodness of fit of the matching can be established by estimating the Mean Square Error in-sample in the estimation window, or out-of-sample by splitting the estimation window into a training $\left[T_{1}, T_{2}\right)$ and a testing window $\left[T_{2}, T_{3}\right]$ (figure B.2 ). In traditional 
events studies goodness of fit is not explicitly mentioned, although inference on the cumulative abnormal returns in the estimation window is considered. The effect of the intervention is equivalent to the abnormal returns of the asset of interest,

$$
\hat{\alpha}_{1, t}=A R_{1, t}=R_{1, t}-R_{1, t}^{N}=R_{1, t}-\sum_{j=2}^{J} w_{j}^{*} R_{j, t}, t \in\left[T_{4}, T_{5}\right]
$$

The optimization problem formulated in expression 4 provides a initial proposal for a a synthetic matching technique. However, this approach is only feasible if the the number of stocks in the control group are of moderate size with respect to the size of the estimation window (the number of pretreatment outcomes) $J<<T_{3}-T_{1}$. In other words we need sufficient time series observations to be able to estimate the $J$-dimensional vector of portfolio weights. If the requirement is not met then we have overdetermined system, more weights than observations. In addition, even if the size of the control group of stocks is reasonable the un-restricted optimization problem could favor solutions where there is larger extrapolation effect in the tracking performance of the portfolio than we would desire. [8] already mention the risk of excessive extrapolation when using synthetic control method. The authors suggest that the optimization problem used to find the optimal weights incorporate restrictions so as to avoid excessive extrapolation. Therefore in their method they restrict to non-negative weights and that the weights have to sum to one. In addition they use exogenous variables for the treated variable and the control group variables so as to match the behavior of the unit of interest to the behavior of the control set not only on the dimension of the variable of interest but also on the other exogenous variables. This approach has some similarities the propensity score based matching as a way to balance estimated causal effect ([5]). Although, synthetic control methods could provide a solution to the extrapolation problem we still have to deal with the large dimensional problem that we face when we use a high dimensional asset space for the control group. This is precisely the case when we look at event studies in the US stock market where we have historical information on more than 5,000 stocks for building a control group.

High-dimensional problems are not new in portfolio optimization an there a couple of techniques that we explore to deal with both the high-dimensional issues but also the extrapolation problems.

Traditional optimal portfolio problems are formulated explicitly using the 
trade-off between return and risk, that is the mean-variance problem $([20])$.

$$
\begin{array}{ll}
\underset{w}{\operatorname{minimize}} & \frac{1}{2} V_{t}\left[\mathbf{R}_{p, t+1}\right]=\mathbf{w}^{\prime} \Sigma \mathbf{w}, \\
\text { subject to } & E_{t}\left[R_{p, t+1}\right]=w^{\prime} \mu=\mu_{p}
\end{array}
$$

where $\mu_{p}$ is the target expected portfolio return and $\Sigma$ is the variance covariance matrix of the universe of expected returns, $\mu$. The optimization problem has a tractable analytical solution

$$
\mathbf{w}^{*}=\frac{\mu_{p}}{\mu^{\prime} \Sigma^{-1} \mu} \Sigma^{-1} \mu
$$

Well documented ill-posed problems arise when we plug-in the sample counterparts of $\mu$ and $\Sigma$, for medium to large size problems in terms of the number of assets considered. Many regularization techniques have been proposed to deal with this problem ([21]; [22]; [23]; [24]). The use of regularization techniques also point to different ways to writing up the optimization problem that will be specially useful to the synthetic portfolio framework ([25]). We can write the variance covariance matrix as the outer product of the returns, and squared first moment, $\Sigma=E\left[\mathbf{R}_{t} \mathbf{R}_{t}^{\prime}\right]-\mu \mu^{\prime}$. Then the empirical counterpart of the expectation of the mean-variance problem is equivalent to the sample mean of the squared $l_{2}$ norm,

$$
\begin{array}{rr}
\underset{w}{\operatorname{minimize}} & E\left[\left|\mu_{p}-\mathbf{w}^{\prime} \mathbf{R}\right|^{2}\right] \\
& \frac{1}{T}\left\|\mu_{p} \mathbf{1}_{T}-w^{\prime} R\right\|_{2}^{2} \\
\text { subject to } & E_{t}\left[R_{p, t+1}\right]=w^{\prime} \mu=\mu_{p}
\end{array}
$$

This same setup can be used to find a solution for the optimal tracking problem as a special case of the optimal portfolio problem where instead of targeting a particular return (scalar) for the porfolio $\mu_{p}$ we are interested in tracking over time a particular stock, in our case the first stock in the asset space, $R_{1, t}$. Let $\mu_{p} \mathbf{1}_{T}:=R_{1, t}$ and $\mathbf{R}:=\left[R_{2, t}, \ldots, R_{J, t}\right]$ denote the subspace of assets that excludes asset 1 , then we get the optimal tracking problem in expression 4 . This optimization problem is not very different form minimizing the sums of square residuals if we let, $Y:=R_{1, t}$ and $X^{\prime} \beta:=\mathbf{R}_{\forall j \neq 1}^{\prime} w$, then we can use ordinary least squares to obtain the portfolio weights. This is a first step toward one solution to the high-dimensional problem by applying regularization to the optimization problem. The least absolute shrinkage and 
selection operator (LASSO) regularization technique introduced by [26] is the $l_{1}$-penalized version of the optimal tracking problem that gives the solution to the synthetic portfolio. The Lasso regularized solution is obtained by solving,

$$
\underset{w}{\operatorname{minimize}} \sum_{t=T_{1}}^{T_{3}}\left(R_{1, t}-\sum_{j=2}^{J} w_{j} R_{j, t}\right)^{2}+\tau \sum_{j=2}^{J}\left|w_{j}\right|
$$

The optimization problem for LASSO provides a long-only portfolios $w_{i} \geq 0$ or impose an specific penalty $(\tau)$ only in the short positions (Brodie et al. 2009). This solution has been recently explored in [11] for a generalization of synthetic control methods. The authors propose the use of LASSO and Elastic net as a way to generalize synthetic control methods. This generalization looks into regularization techniques as a way to improve on the original approach proposed by [8] both in terms on the restrictions on the weights and the introduction of exogenous covariates. The authors propose a class of estimators that can be used depending on the size of the estimation window $T_{3}-T_{1}$ (the number of pre-treatment outcomes) and the number of stocks in the control group $J$. In other words theses estimators leverage the use of regularization methods and restrictions in the optimization/estimation problem to deal with the challenges of a high-dimensional problem. They proposed an elastic net type penalty for regularization,

$$
\underset{w}{\operatorname{minimize}} \sum_{t=T_{1}}^{T_{3}}\left(R_{1, t}-\sum_{j=2}^{J} w_{j} R_{j, t}\right)^{2}+\tau\left(\frac{1-\alpha}{2} \sum_{j=2}^{J} w_{j}^{2}+\alpha \sum_{j=2}^{J}\left|w_{j}\right|\right)
$$

The elastic net is a regularized regression method that linearly combines the $l_{1}$ and $l_{2}$ penalties of the lasso and ridge methods. In addition to the penalty $\tau$ we introduce a parameter for the optimal linear combination $\alpha$. Therefore, in this case be have two tunning parameters $(\tau, \alpha)$. The authors illustrate the methods using the data for three seminal studies in causal inference.

Finally, since in portfolio optimization it is important to compare any methods to a computationally inexpensive benchmark we can define a naive synthetic portfolio as the solution to the optimization problem where the weights are constant (and sum up to 1) across the members in the control group, $w_{j}=\bar{w}:=\frac{1}{J-1}, j=2, \ldots, J$. The effect of the intervention is the 
(naive) abnormal returns,

$$
\hat{\alpha}_{1, t}^{\bar{w}}=A R_{1, t}=R_{1, t}-R_{1, t}^{\bar{w}}=R_{1, t}-\frac{1}{J-1} \sum_{j=2}^{J} R_{j, t}, t \in\left[T_{4}, T_{5}\right]
$$

The naive synthetic portfolio is the cross sectional simple average of all of the available stock that are not affected by the event. The naive synthetic portfolio can be seen as a special case of the difference-in-difference method (DID). The average cumulative abnormal returns is the simple time average of the estimated abnormal returns over the event window for the firm affected by the event, $A C A R_{1}=\frac{1}{T_{5}-T_{4}} \sum_{t=T_{4}}^{T_{5}} \hat{A R_{1, t}}{ }^{6}$. In the DID method the average cumulative abnormal returns for firm 1 (over the event window) is a function of different time series and cross sectional averages over the the returns of firm 1 and the returns of the control group in the estimation and event windows. Let $D_{1}$ denote the difference in the returns before and after the event,

$$
D_{1}=\frac{1}{T_{5}-T_{4}} \sum_{t=T_{4}}^{T_{5}} R_{1, t}-\frac{1}{T_{3}-T_{1}} \sum_{t=T_{1}}^{T_{3}} R_{1, t}
$$

It is important to note that expression 12 is equivalent to the constant mean model in the traditional event study literature. Let $D_{c o}$ denote the difference in the returns before and after the event (where every element in the control group has an equal weight) for the control group,

$$
D_{c o}=\frac{1}{J-1} \sum_{j=2}^{J}\left[\frac{1}{T_{5}-T_{4}} \sum_{t=T_{4}}^{T_{5}} R_{j, t}-\frac{1}{T_{3}-T_{1}} \sum_{t=T_{1}}^{T_{3}} R_{j, t}\right] .
$$

If we denote $\bar{R}_{1,\left(T_{1}, T_{3}\right)}=\frac{1}{T_{3}-T_{1}} \sum_{t=T_{1}}^{T_{3}} R_{1, t}$ and $\bar{R}_{c o,\left(T_{1}, T_{3}\right)}=\frac{1}{T_{3}-T_{1}} \sum_{t=T_{1}}^{T_{3}}\left[\frac{1}{J-1} \sum_{j=2}^{J} R_{j, t}\right]$ as the average returns during the estimation window for firm 1 and the control group (taking a simple average over the cross-section), respectively. Then we can show that the DID average treatment effect using the Difference in

\footnotetext{
${ }^{6}$ In order to avoid confusion it is important to note that in the traditional event study literature the average cumulative abnormal returns the average is over the cross-section of firms affected by the event. This is the main object of interest in applications to determine if a particular event has an impact on the value of the firm. For the moment we deviate from that purpose because we only consider the event on one firm.
} 
Difference method is,

$$
\begin{aligned}
A C A R_{1}^{D I D} & =\frac{1}{T_{5}-T_{4}} \sum_{t=T_{4}}^{T_{5}}\left[R_{1, t}-R_{1, t}^{\bar{w}}\right]-\left(\bar{R}_{1,\left(T_{1}, T_{3}\right)}-\bar{R}_{c o,\left(T_{1}, T_{3}\right)}\right) \\
& =\frac{1}{T_{5}-T_{4}} \sum_{t=T_{4}}^{T_{5}}\left[R_{1, t}-\frac{1}{J-1} \sum_{j=2}^{J} R_{j, t}\right]-\left(\bar{R}_{1,\left(T_{1}, T_{3}\right)}-\bar{R}_{c o,\left(T_{1}, T_{3}\right)}\right)
\end{aligned}
$$

This implies that the DID estimator is equivalent to the naive synthetic portfolio estimator with an adjustment to the average return difference between the treated and the control group in the estimation window. This adjustment acts as an intercept correction for the forecast in the event window.

To test for the statistical significance of the average treatment effect over the event window we use the end-of-sample instability test of [27] following recent work by [13]. [13] provides tests statistics and the distribution for average treatment effects estimated by synthetic control methods for two different cases based on the relative size of the events window with respect to the estimation window. Since, short term financial event studies have a small event window $(-1,1)$ we can use the tests statistics provided by [13] for synthetic control methods. Recall that in expression 5 we noted that the treatment effect on firm 1 is equivalent to the abnormal return of firm 1 , $A R_{1, t}$; taking the average over the event window we can obtain the average treatment effect,

$$
A A R_{1}=\frac{1}{T_{5}-T_{4}} \sum_{t=T_{4}}^{T_{5}} A R_{1, t}=\frac{1}{T_{5}-T_{4}} \sum_{t=T_{4}}^{T_{5}} R_{1, t}-\hat{R}_{1, t}^{N} .
$$

Recall that $\hat{R}_{1, t}^{N}$ is estimated using the market model or the synthetic portfolio weights. We want to test the null hypothesis, $H_{0}: A R_{1, t}=A R_{1,0}$ for all $t=T_{4}, \ldots, T_{5}$, against a strictly positive treatment effect alternative, $H_{a}$ : $A A R_{1}=E\left[A R_{1, t}\right]>A R_{1,0}$. The test statistic for such a test,

$$
\hat{B}_{T_{5}-T_{4}}=\frac{1}{\sqrt{T_{5}-T_{4}}} \sum_{t=T_{4}}^{T_{5}} R_{1, t}-\hat{R}_{1, t}^{N}-A R_{1,0}
$$

We derive the empirical distribution of the statistic by block sampling the estimation and gap window using the length of the event window; first we 
compute,

$\hat{B}_{T_{5}-T_{4}, j}:=\frac{1}{\sqrt{T_{5}-T_{4}}} \sum_{t=j}^{T_{5}-T_{4}+j-1} R_{1, t}-\hat{R}_{1, t}^{N}$, for $j=1, \ldots, T_{4}+1-\left(T_{5}-T_{4}\right)$.

As mentioned in [13], the empirical distribution of $\left[\hat{B}_{T_{5}-T_{4}, j}\right]_{j=1}^{T_{4}+1-\left(T_{5}-T_{4}\right)}$ can be used to obtain critical values for the test statistic $\hat{B}_{T_{5}-T_{4}}$ under the null hypothesis. If $\hat{B}_{T_{5}-T_{4}}$ is at the tail of the empirical distribution then we reject the null hypothesis, $H_{0}: A R_{1, t}=A R_{1,0}$ for all $t=T_{4}, \ldots, T_{5}$. This hypothesis test and the statistic is only valid for treatment effects that are constant across the event window, note that this mights be true for short term event studies but no necessarily for long term event studies. The main advantage of the test is that by considering the event as an structural change in the end of the sample then it is possible to use the abundant information in the estimation window to obtain re-sampled values of the statistic and perform inference. One of the biggest obstacles that the traditional framework of short term events studies faces is the very few observations available in the event window for individual events. With these few number of observations (3,11 or 21 days) individual test on abnormal returns have such small degrees of freedom such that they are hardly ever considered; therefore most of the reported inference is based on pooling the events. Andrew's end-of-sample stability test provides a simple framework to overcome this problem in financial event studies. As far as we are aware of although Andrews test is not specifically designed with in the framework the causal inference literature we find that it has not been previously considered as a viable alternative to individual test on average cumulative abnormal returns, [3] and [1].

\section{Simulation}

In the previous sections we provide an overview of traditional event studies and four alternative methods for causal events studies in finance based on the synthetic portfolio approach and its relationship to DID in this context. We mention in the introduction the importance of a causal approach to event studies, but in addition these alternative methods are able to handle the high-dimensional challenge brought by the large asset space in the US stock market. The main reason that we want to consider a large asset space is to provide a matching technique that avoids any $A d$ hoc choice of which 
stock should be in the control group. This is a desired property since the abnormal returns (estimated effects of the intervention) will be more robust and less sensitive to the choice of the researcher. These regularized estimators have the advantage that they provide automatic punning of the units within the control group and hence they avoid any manipulation in favor of a particular hypothesis; this is desired property of the estimator and the research design $([28])$.

From a statistical point we can test the viability of the alternative methods by looking at the biased and variance of the proposed estimators for the average treatment effects. In addition, we measure the mean square error of the synthetic portfolio in-sample and out-off-sample in the estimation window. For this reason split the estimation window into a training and testing window (B.2). Although we have argued that with the synthetic portfolio approach there could be less model risk than accepting the market model to build the potential outcome, there could potentially be the risk of overfitting by the best synthetic tracking portfolio. The mean square error and the estimated treatments effect will give us an idea of the possible trade-off regarding over-fitting, bias and variance of the proposed estimators.

We now setup a simulation exercise in order to determine the statistical and financial merits of the alternative methods for event studies. The simulation considers a large dimensional asset space of five hundred assets $J=500$. We consider a short term financial event study where cumulative abnormal returns are measured one day before and after the event $(-1,1)$ for the stock return that is affected by the event, $R_{1, t}$. In event studies with daily data the estimation window has a length of 150 trading days. We consider these 150 trading days as the training data and introduce 50 trading days for the testing window. In addition we consider a gap window with a duration of 25 trading days. Therefore the training window $\left[T_{1}, T_{2}\right)$ will cover trading days $[-226,-76)$ and the testing window will cover trading days $[-76,-27)$. We perform 10, 000 simulations.

The data generating process for all of the stocks is a one-factor model (a CAPM model with estimated $\beta$ 's) or a stationary first order autoregressive process. For the one factor model,

$$
R_{i, t}=\alpha+\beta R_{m, t}+\varepsilon_{i, t},
$$

we first simulate the market return $R_{m, t} \sim N(0,1)$, fix a value for $\alpha=0.1$ and set a value for $\beta=0$ for the first hundred stocks, for $\beta=0.2$ for the next hundred stocks and so on until the last hundred assets with $\beta=1$. The 
idiosyncratic component for each stock is simulated as, $\varepsilon_{i, t} \sim N(0,1.5)$. We use this data generating process to simulate the behavior of all of the stocks and during the entire time line. However, for the stock 1 affected by the event we simulate the effect of the event at the event time $T_{4}+1$ such that, $R_{1, t}^{N}=R_{1, t}$ for $t>T_{4}$ is the latent potential outcome, in other words the realization where the event does not take place. The observed outcome (the event takes place) is given by $R_{1, T_{4}+1}=R_{1, t}+\gamma$, where $\gamma=-0.035$ implies a drop in the returns of $3.5 \%$ once the event takes place, for example a merger announcement.

For the first order autoregressive process,

$$
R_{i, t}=\alpha+\phi R_{i, t-1}+\varepsilon_{i, t}
$$

we simulate the idiosyncratic component $\varepsilon_{i, t}$ as we did in the previous case and start the recursion with $R_{i, 0}=0$. We consider different values for $\phi$ consistent with stationarity and $\alpha=0.1$. The effect of the event on stock 1 is modeled as in the previous case. An important difference from the previous case is that we do not have an explicit simulation of the market returns $R_{m, t}$, required to estimate the market model. In this case we use as the market return the ex-post equal weighted portfolio (excluding the stock 1), $R_{m, t}:=\frac{1}{J-1} \sum_{j=2}^{J} R_{j, t}$. After simulating the returns using each of the data generating process we estimate the abnormal return at the event date $T_{4}+1$ which is equivalent to the treatment effect, $\hat{\gamma}$. We look at the average treatment effect over all the simulations and compare across the different models.

The results are summarized in tables A.1, A.2 and A.3. The mean square error in the training and testing window indicates that there is some degree of over-fitting in synthetic portfolio method, specially using Elastic-Net. The overall performance of the Elastic-Net estimator is above all other estimators in the training window but not in the testing window. On the other hand the performance for the naive synthetic and the difference-in-difference estimator is rather stable at both in and out-of-sample. The Market model also shows good performance, specially when the the asset of interest $R_{1, t}$ has a true $\beta$ that approaches one $(\beta=0.8$ and $\beta=1)$. This is to be expected because this is the situation where the market model provides the best tracking performance. When the data generating process is an autoregressive model and the process has a strong autocorrelation the performance of all of the methods is rather poor.

In table A.3 we look at the estimated treatment effect and the variance (in 
parenthesis). The true treatment effect is $\gamma=-0.035$ and hence we are interested in the performance of the estimators that indicate the lowest bias. The lowest bias is obtained by the Elastic-Net estimators for $\beta=0.5,0.8$, the synthetic naive estimator for $\beta=0.2$ and the market model for $\beta=1$. With respect to the variance all of the estimators indicate a large uncertainty around the point estimates and it is not clear which is preferable in terms of the variance, therefore relative performance can only be based on the bias. When the data generating process is an autoregressive model with either a strong persistence or driven only by noise performance of all of the estimators is poor.

\section{Empirical application: Merger announcements}

We obtain M\&A data from the Thomson Reuters SDC Platinum Financial Securities database. Thomson Reuters SDC collects all M\&A transactions in the US that involve at least $5 \%$ of the ownership change of a company. We apply several filters to the M\&A data that are common practice in the literature [29]. We download all US M\&A transactions from 2003 to 2014. After applying these filters and merging the resulting events with the CRSP databases, we identify 5,025 M\&A announcements from June 2003 to December 2014.

The theoretical and empirical literature on the effects of merger announcements is extensive and our aim is not to provide an exhaustive overview (there are some very comprehensive reviews, [30] more recently an on-line special issue of financial management).

We focus on identifying the main empirical results and the well established empirical regularities that have been published using predominantly traditional event study methodologies with a large scale sample, that it where the number of events exceeds 1,000 observations. We also look at the more recent literature that has used propensity score matching as a first step toward balancing the treatment and control groups, in particular, [7] and [31]

Table A.4 provides a short summary of the effects of mergers on market value of the firm. This summary is meant to be illustrative rather than comprehensive. One important observation is that most of these large scale studies have been performed with data from the 80's and 90's. The literature is concerned not only in a brought measure of impact for all firms but in a quest to determine how theses effects change along different dimensions, for 
example by taking the point of view of the acquirer/bidder or the target, whether the company is publicly traded or a private company, if the merger is financed using cash or stock, or if there is a diversification motive from the bidder $^{7}$. Some of the empirical regularities are: positive (negative) effects are observed for bidders acquiring private (public) firms; positive effects are observed for target firms when the acquierer is either a public or a private firm, but the value creation is larger for the former; all stock (all cash) financed bids have a negative (positive) effect on stock performance, unless the target is a private firm where stock finances acquisitions create value. The more recent literature has also taken advantage of propensity score matching to rebalanced the sample of teated and control firms, in the first example all stock finances acquisitions still create negative effects as apposed to all cash deal, however by using propensity score matching the difference between the effects for all-cash or all-stocks is smaller. Finally, the observed premium for target public firms versus private firms becomes statistically not significant by after using propensity score.

We examine the effects of merger announcements of the firm value in the short term using the competing method, but we reports the findings that used the traditional method (the market model), synthetic portfolio (where estimation is base on Elastic-net version with non-negative portfolio weights), and difference-in-difference (we have shown in section 3 that is related to the naive synthetic portfolio.). We also estimates these effects along different subsets of data that have been well studies in the literature, A.4.

The results are presented in figure ??. The average estimated effects are nominally very similar across the different methodologies. It is important to note that these average are estimated using the cross section of events, and although the averages are similar in magnitude, we reject the null hypothesis that the sample of individual measures are statistically equivalent across the different methodologies. These results are consistent with the simulations where we find close estimates of the true treatment across the different methodologies. The sample specific results are consistent with the literature, for example the effect on the bidders performance is small varying form

\footnotetext{
${ }^{7}$ This diversification motive takes into account if the target firm belong to a significantly distant industry from the bidding firm.
} 
$0.52 \%$ to $0.71 \%$ and for the target the it is quite large varying from $19.4 \%$ to $20 \%$. In the literature, based on an earlier historical sample, these estimates are around $1.8 \%$ and $27.7 \%$, respectively. We also observe the change in the sign, for a bidder acquiring a private firm $(0.78 \%$ to $1 \%)$ versus a public firm $(-0.67 \%$ to $-0.57 \%)$. On the other hand with all of the three methods we do not observe that $100 \%$ stock financed mergers has a negative affect on the bidder after the announcement, the effect is positive $(0.27 \%$ to $0.38 \%)$ but small than in the case of $100 \%$ cash deals $(0.5 \%$ to $0.66 \%)$. For all other cases such as diversified/un-diversified, a single or more than one bidder the signs and the magnitude are within the range of the results in the literature.

As mentioned in 3 one of the additional benefits of framing traditional event studies within causal inference is the use of Andrews end-of-sample instability test. This test is not affected by the extremely small sample of the event window that makes traditional parametric t-test of non-parametric wilcoxon test, difficult to apply in this context. Therefore we can use the subset of events where we can reject the null hypothesis that the treatment effect is zero and therefore we have events either with strictly positive or negative effects of the merger. That is we are interested in the number of cases where the effects is different form zero, these samples represent anywhere from $8 \%$ to $25 \%$ of the total sample, for example out of 1,009 events for acquisitions where the announcement involves a public target, there are 144 cases where the effect on the bidder is significantly positive and 223 cases where it is significantly negative. This means that overall negative average effect is strongly affected by a number of treatment effects for approximately 640 cases where the true effect is statistically non-existent. In figure B.3 we use the sub-samples to get an idea of the magnitude of the effects along the different cases considered in the literature. This provides a way to derive some lower and upper bound for the treatment effect that circumvents that controls for the individual events for which the true effect of the merger announcement is zero. The magnitude of these effects are more or less equivalent across the different methodologies therefore we only report the results based on the traditional market model. The results indicate, that although as we saw before that the overall average effect of merger announcements is negative on the firm value of the bidder if the target is a public company, there are 146 events in the sample where the effect is in average $8.4 \%$. Another interesting case is for $100 \%$ stock financed mergers, where the literature reports negative effects on the bidder in the magnitude of $-3.5 \%$, and we find an overall small 
but positive effect $0.37 \%$ and also a subset of firms where the average effect is significantly positive $16 \%$ and significantly negative $-9.1 \%$.

\section{Conclusions and future research}

The methodological framework for events studies was laid out in the late sixties and although the testing framework has evolved, the estimation approach has not change profoundly since it was laid out. Research on methods has also received little attention and even though the estimation of cumulative abnormal returns is not the main focus of the papers in empirical finance they still represent an important first in many empirical motivated research questions across the different areas of finance. In the last twenty years research in causal inference methods has introduced more credible tools to re-examine causal effects that are at the core of many questions that motivate empirical research. This paper provides a simple unifying framework between traditional event study estimation methods and modern causal inference methods, in particular synthetic control methods. The framework is based on common tool in finance which is an optimal tracking portfolio. Although causal inference methods are already within the toolkit of empirical finance researchers, in particular the use of propensity score matching, a competing method like synthetic control has not been extensively used. The simulation exercise and the empirical application indicate that the market model provides a counterfactual as good as the synthetic portfolio estimators we introduce. Fitting the return of interest through the market model is also based on the idea that a portfolio provides a good potential outcome, therefore the results are not too surprising. Additional research will eventually tell us if the conditions under which this performance is adequate hold in general or under specific conditions for example if the asset space for estimating these portfolios is sufficiently large and/or the granularity of the elements in the portfolio.

\section{Acknowledgements}

This research was completed while the author was visiting the finance department in the business school of the University of Connecticut, the author would like to thank Chinmoy Ghosh for the invitation. The paper benefited from the comments received from Jose Martinez and Cristian Pinto and seminar participants at the business school and the actuarial science group at the department of mathematics in the University of Connecticut. 


\section{References}

[1] S. Kothari, J. Warner, Handbook of Corporate Finance: Empirical Corporate Finance, Elsevier, North-Holland, pp. 3-36.

[2] R. Ball, P. Brown, An empirical evaluation of accounting income numbers, Journal of Accounting Research 6 (1968) 159-178.

[3] J. C. Corrado, Event studies: A methodology review, Accounting and Finance 51 (2011) 207-234.

[4] I. Gow, D. Larcker, P. Reiss, Causal inference in accounting research, Journal of Accounting Research 54 (2016) 477-523.

[5] G. Imbens, D. Rubin, Causal inference for statistics, social and biomedical sciences: an introduction, Cambridge University Press, 2015.

[6] X. Li, X. Zhao, Propensity score matching and abnormal perfomence after seasoned equity offerings, Journal of Empirical Finance 13 (2006) $351-370$.

[7] M. Svetina, Managerial motives in mergers: Propensity score matching approach, Manegerial and Decision Economics 33 (2012) 537-547.

[8] A. Abadie, A. Diamond, J. Hainmuller, Synthetic control methods for comparative case studies: Estimating the effect of californias tobacco control program, Journal of the American Statistical Association 105 (2010) 493-505.

[9] S. Athey, G. Imbens, The state of applied econometrics: causality and policy evaluation, Journal of Economic Perspectives 31 (2017) 3-32.

[10] J. Xu, Genralized synthetic control method: causal inference with interactive fixed effects models, Political Analysis 25 (2017) 57-76.

[11] N. Doudchenko, G. Imbens, Balancing, regression, difference-indifference and synthetic control methods: A synthesis, 2017.

[12] B. Ferman, C. Pinto, Inference in differences-in-differences with few treated groups and heteroscedasticity, 2017. 
[13] K. Li, Statistical inference for average treatment effects estimated by synthetic control methods, 2017.

[14] C. Carvalho, R. Masini, M. Medeiros, Arco: An artificial counterfactual approach for high-dimensional panel time series data, 2016.

[15] D. Acemoglu, S. Johnson, A. Kermani, A. Kwak, T. Mitton, The value of connections in turbulent times: Evidence from the united states, Journal of Financial Economics 121 (2016) 368-391.

[16] C. Castro, D. Agudelo, S. Preciado, Measuring the effectiveness of volatility auctions, 2017.

[17] M. Mitchell, J. Netter, The role of financial economics in securities fraud cases: applications at the securities and exchange commission, Business Lawyer 49 (1994) 545-590.

[18] C. Harvey, Y. Liu, H. Zhu, and the cross-section of expected returns, The Review of Financial Studies 29 (2016) 5-68.

[19] A. MacKinlay, Event studies in economics and finance, Journal of Economic Literature 35 (1997) 13-39.

[20] H. Markovitz, Portfolio selection, The Journal of Finance 7 (1952) $77-91$.

[21] P. A. Frost, J. E. Savarino, An empirical bayes approach to efficient portfolio selection, The Journal of Financial and Quantitative Analysis 21 (1986) 293-305.

[22] O. Ledoit, M. Wolf, Honey, i shrunk the sample covariance matrix, The Journal of Portfolio Management 30 (2004) 110-119.

[23] J. Tu, G. Zhou, Markowitz meets talmud: A combination of sophisticated and naive diversification strategies, Journal of Financial Economics 99 (2011) 204-215.

[24] J. Brodie, I. Daubechies, C. De Mol, D. Giannone, I. Loris, Sparse and stable markowitz portfolios, PNAS 106 (2009) 12267-12272.

[25] M. Carrasco, N. Noumon, Optimal portfolio selection using regularization, 2010. 
[26] R. Tibshirani, Regression shrinkage and selection via the lasso, Journal of the Royal Statistical Society. Series B (Methodological) 58 (1996) $267-288$.

[27] D. Andrews, End-of-sample instability test, Econometrica 71 (2003) 1661-1694.

[28] G. King, R. Nielsen, Why propensity scores should not be used for matching, 2016.

[29] H. Mulherin, A. Simsir, Measuring deal premiums in takeovers, Financial Management Spring (2015) 1-14.

[30] B. Eckbo, Bidding strategies and takeover premiums: a review, Journal of Corporate Finance 15 (2009) 149-178.

[31] A. Golubov, D. Petmezas, N. Travlos, Do stock-financed acquisition destroy value? methods and evidence, Review of Finance (2016) 161200. 
DGP: 1 factor model

DGP: Autoregressive model

\begin{tabular}{lcccccc} 
Estimator & $\beta=0.2$ & $\beta=0.5$ & $\beta=0.8$ & $\beta=1$ & $\phi=0$ & $\phi=0.9$ \\
\hline Market & 8.89 & 8.88 & 8.88 & 8.88 & 12.18 & 57.20 \\
Syn.Lasso & 8.78 & 8.72 & 8.68 & 8.64 & 9.21 & 5.98 \\
Syn.Elastic-Net & 6.84 & 6.52 & 6.24 & 6.07 & 5.27 & 3.74 \\
Syn.Elastic-Net $\$ \%{ }^{*} \$$ & 6.86 & 6.55 & 6.26 & 6.09 & 5.57 & 6.05 \\
Syn.naive & 9.12 & 9.02 & 9.11 & 9.27 & 12.49 & 66.19 \\
Dif-in-Dif & 9.12 & 9.02 & 9.11 & 9.27 & 12.49 & 66.19 \\
\hline
\end{tabular}

Note: The mean square error is estimated in-sample using the information in the testing subset of data from the estimation window, $t \in\left[T_{1}, T_{2}\right)$. All of the estimators that use regularization impose non-negative weights. The estimator Syn.Elastic-Net* includes a constant in the estimation of the weights.

Table A.1: Mean square error in training window

\section{Appendix A. Tables}


DGP: 1 factor model

DGP: Autoregressive model

\begin{tabular}{lcccccc} 
Estimator & $\beta=0.2$ & $\beta=0.5$ & $\beta=0.8$ & $\beta=1$ & $\phi=0$ & $\phi=0.9$ \\
\hline Market & 9.11 & 9.12 & 9.10 & 9.09 & 11.01 & 17.83 \\
Syn.Lasso & 9.05 & 9.23 & 9.49 & 9.72 & 9.81 & 38.59 \\
Syn.Elastic-Net & 9.40 & 9.47 & 9.57 & 9.70 & 9.69 & 68.01 \\
Syn.Elastic-Net* & 9.39 & 9.47 & 9.57 & 9.70 & 9.69 & 60.81 \\
Syn.naive & 9.10 & 9.01 & 9.09 & 9.24 & 10.06 & 21.47 \\
Dif-in-Dif & 9.10 & 9.01 & 9.09 & 9.24 & 10.06 & 21.47 \\
\hline
\end{tabular}

Note: The mean square error is estimated out-of-sample using the information in the testing subset of data from the estimation window, $t \in\left[T_{2}, T_{3}\right)$. All of the estimators that use regularization impose non-negative weights. The estimator Syn.Elastic-Net* includes a constant in the estimation of the weights.

Table A.2: Mean square error in testing window

DGP: 1 factor model

DGP: Autoregressive model

\begin{tabular}{lcccccc} 
Estimator & $\beta=0.2$ & $\beta=0.5$ & $\beta=0.8$ & $\beta=1$ & $\phi=0$ & $\phi=0.9$ \\
\hline Market & -0.030 & -0.053 & -0.028 & -0.056 & 0.001 & -0.001 \\
& $(2.97)$ & $(3.03)$ & $(3.02)$ & $(2.97)$ & $(1.01)$ & $(1.02)$ \\
Syn.Lasso & 0.060 & 0.030 & 0.049 & 0.003 & 0.001 & -0.002 \\
& $(2.96)$ & $(3.06)$ & $(3.09)$ & $(3.07)$ & $(1.00)$ & $(1.08)$ \\
Syn.Elastic-Net & -0.022 & -0.045 & -0.038 & -0.081 & 0.001 & -0.002 \\
& $(3.02)$ & $(3.09)$ & $(3.11)$ & $(3.07)$ & $(1.00)$ & $(1.08)$ \\
Syn.Elastic-Net* & -0.021 & -0.044 & -0.039 & -0.082 & 0.001 & -0.002 \\
& $(3.01)$ & $(3.09)$ & $(3.11)$ & $(3.07)$ & $(1.04)$ & $(1.08)$ \\
Syn.naive & -0.036 & -0.054 & -0.032 & -0.065 & 0.001 & -0.001 \\
& $(2.96)$ & $(3.02)$ & $(3.02)$ & $(2.99)$ & $(1.00)$ & $(1.04)$ \\
Dif-in-Dif & -0.037 & -0.053 & -0.031 & -0.061 & -0.001 & -0.001 \\
& $(2.97)$ & $(3.03)$ & $(3.03)$ & $(3.00)$ & $(1.00)$ & $(1.03)$ \\
\hline
\end{tabular}

Note: Average treatment effects are reported for the simulation exercise along with the variance in parenthesis. The true treatment effect is $\gamma=-0.035$ and the number of simulations is 10,000. All of the estimators that use regularization impose non-negative weights. The estimator Syn.Elastic-Net* includes a constant in the estimation of the weights.

Table A.3: Simulated treatment effect 


\begin{tabular}{|c|c|c|c|c|}
\hline Study & $\mathrm{ACAR} \%$ & samp & and result & \\
\hline $\begin{array}{l}\text { M\&A Announcement-induced ACA } \\
\text { sample (1980-2002) }\end{array}$ & to U.S & Bidde & s, large & \\
\hline & & all & public $^{1}$ & private \\
\hline Fuller, Netter, and Stegemoller (2002) & $(-2,2)$ & 1.80 & -1.00 & 2.10 \\
\hline Bradley and Sundaram (2006) & $(-2,2)$ & 1.40 & -0.70 & 1.90 \\
\hline Betton, Eckbo, and Thorburn (2009) & $(-1,1)$ & -1.20 & & \\
\hline & & & undiv. $^{2}$ & div. \\
\hline Akbulut and Matsusaka (2003) & $(-2,1)$ & & 1.20 & 1.10 \\
\hline & & & stocks $^{3}$ & cash \\
\hline Savor (2006) & $(-1,1)$ & & -3.50 & 1.00 \\
\hline & & & public $^{4}$ & private \\
\hline Moeller, Shlingemann and Stultz (2007) & $(-1,1)$ & 0.80 & -2.30 & 3.40 \\
\hline $\begin{array}{l}\text { M\&A Announcement-induced ACA } \\
\text { sample (1985-2009), Propensity Sco }\end{array}$ & to U.S & Bidde & s, large & \\
\hline & & all & stocks $^{3}$ & cash \\
\hline Golubov, Petmezas, Travlos (2015) & $(-2,-2)$ & -1.31 & -2.29 & 0.50 \\
\hline & & 0.91 & & \\
\hline & & & No $\mathrm{PSM}^{5}$ & PSM \\
\hline diference all-stock, all-cash & & & 2.79 & 1.53 \\
\hline $\begin{array}{l}\text { M\&A Announcement-induced ACA } \\
\text { sample (1980-2002), Propensity Sco }\end{array}$ & to U.S & Targ & , large & \\
\hline & & & public $^{6}$ & private \\
\hline Svetina (2012) & $(-1,1)$ & & 27.79 & 17.69 \\
\hline
\end{tabular}

Note: 1) public and private target firms in the merger. 2) underspecified or diversified deals, meaning that the target firms belongs or does not belong to the same sector. 3) The merger is finance completely by cash or by stocks form the point of view of the acquirer. 4) The target firm is public or private and the acquirer is financing the bid using stocks. 5) propensity score matching is used to balance the treatment and control sample in the estimation of the treatment effects. 6) public and private bidding firms in the merger (in this particular result the difference becomes statistically insignificant once propensity score is used).

Table A.4: Review of the literature 


\section{Appendix B. Figures}

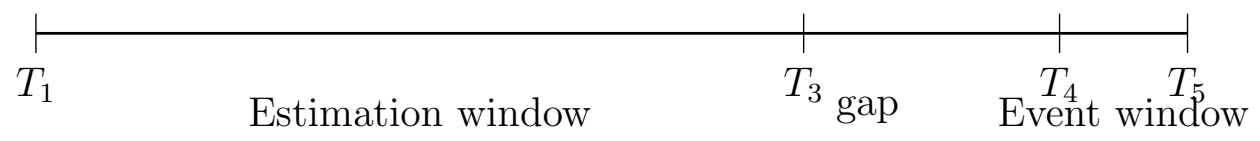

Figure B.1: Timeline for an event study.

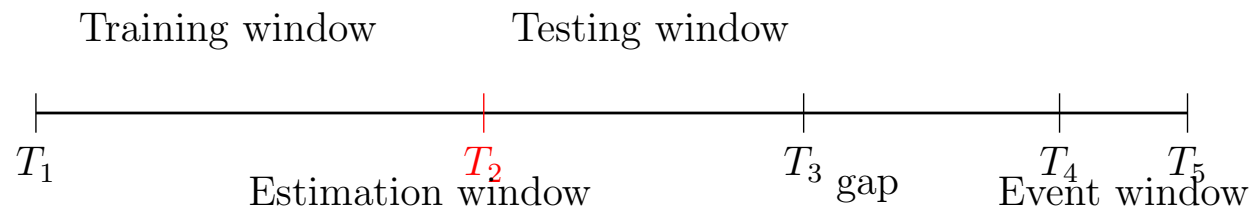

Figure B.2: Timeline for an event study with a training and testing window. 


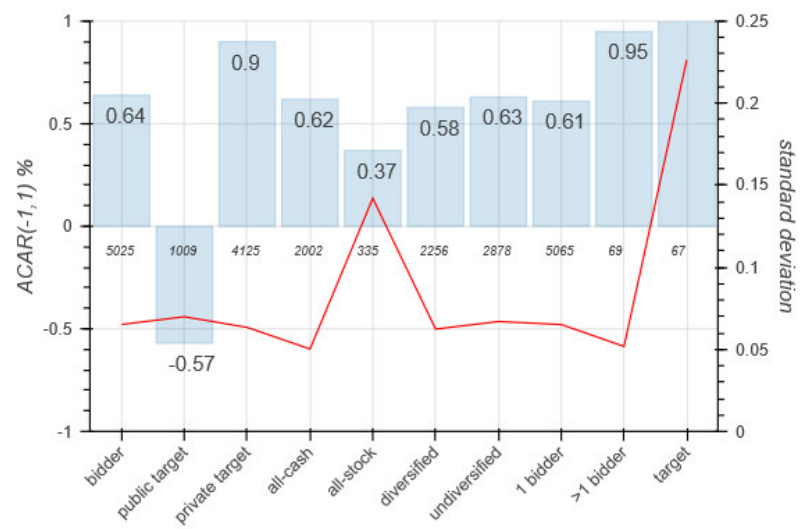

(a) Average cummulative abnormal returns, market model.

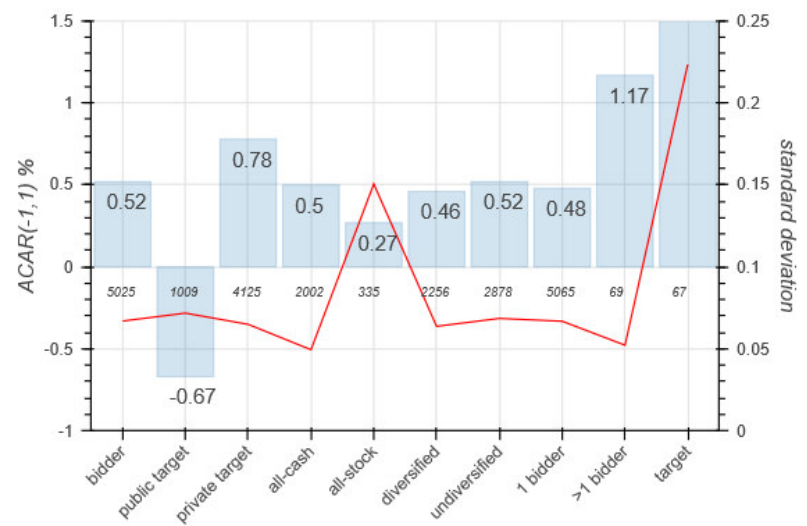

(b) Average cummulative abnormal returns, elastic net.

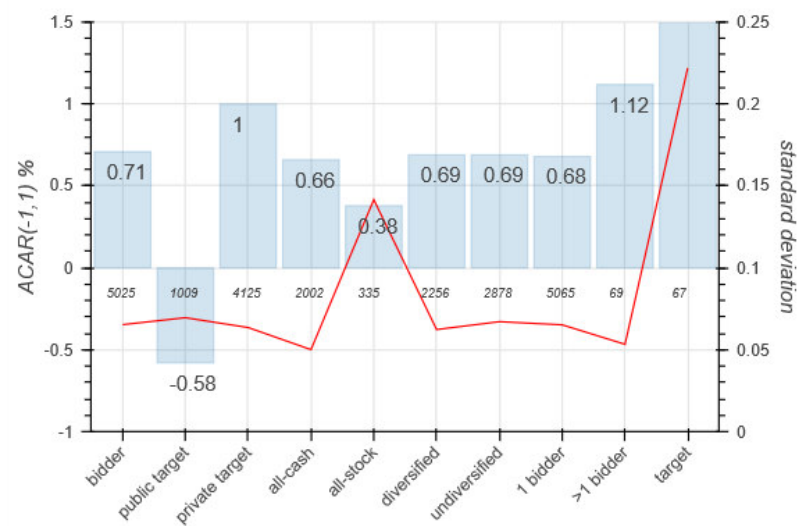

(c) Average cummulative abnormal returns, difference-in-difference. Note: The numbers in italic along the $\mathrm{x}$-axis indicate the number of events that are considered for each case. 


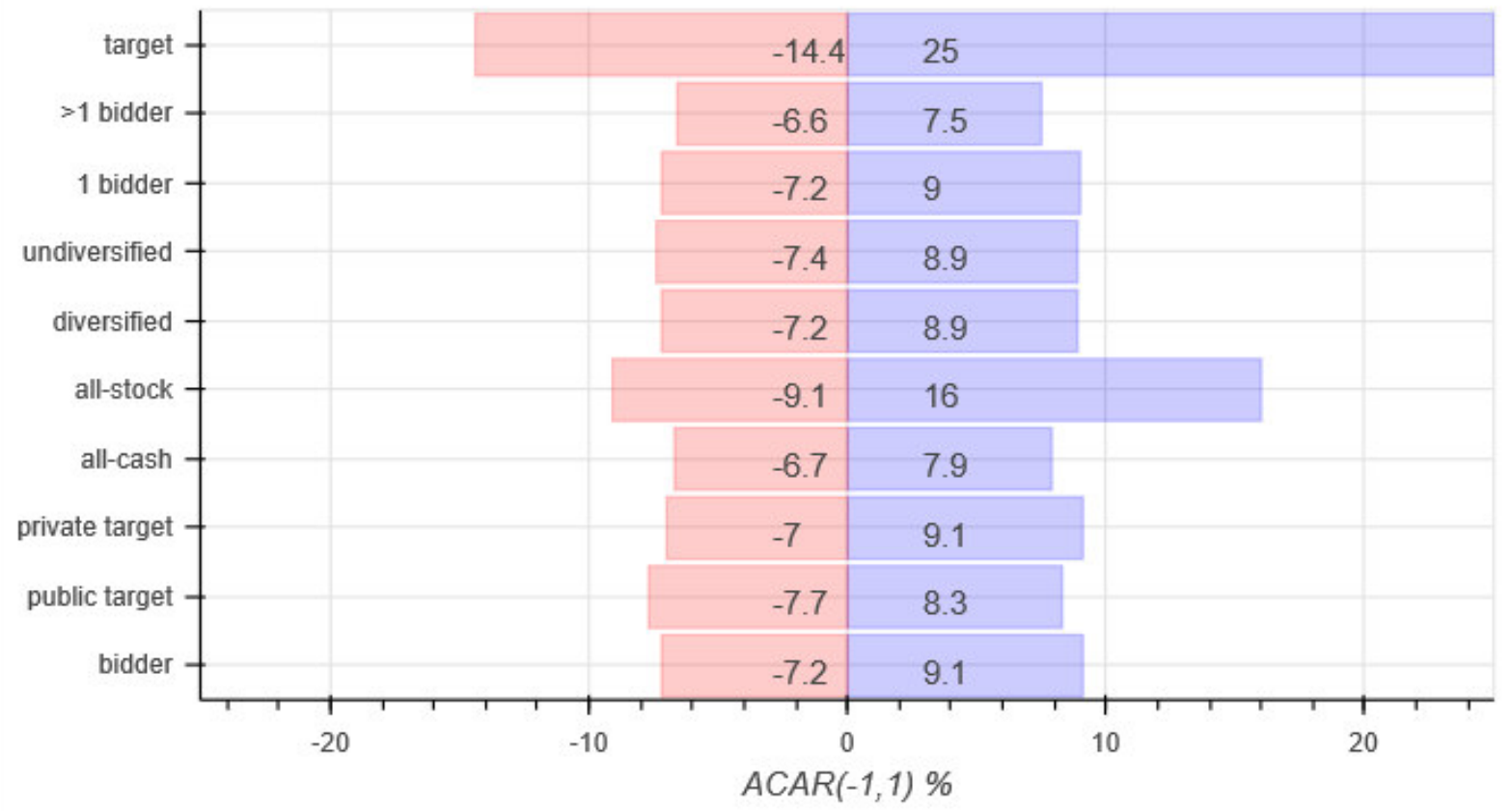

Figure B.3: Average cummulative abnormal returns, for events where the treatment effect is statistically above or below zero, using Andrews end-of-sample stability test. 